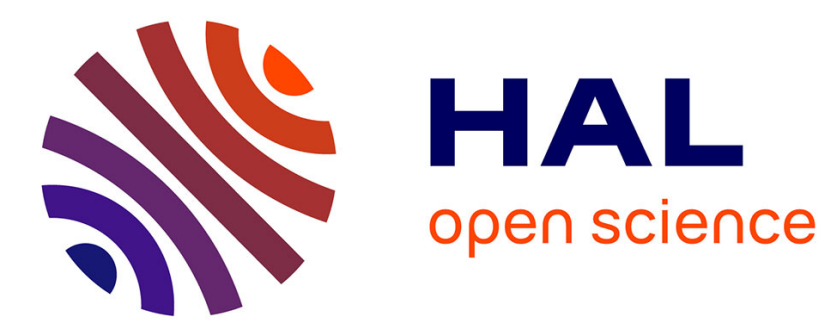

\title{
Electromagnetic properties of the Dayem bridge
}

M. T. Levinsen

\section{To cite this version:}

M. T. Levinsen. Electromagnetic properties of the Dayem bridge. Revue de Physique Appliquée, 1974, 9 (1), pp.135-144. 10.1051/rphysap:0197400901013500 . jpa-00243723

\section{HAL Id: jpa-00243723 https://hal.science/jpa-00243723}

Submitted on 1 Jan 1974

HAL is a multi-disciplinary open access archive for the deposit and dissemination of scientific research documents, whether they are published or not. The documents may come from teaching and research institutions in France or abroad, or from public or private research centers.
L'archive ouverte pluridisciplinaire HAL, est destinée au dépôt et à la diffusion de documents scientifiques de niveau recherche, publiés ou non, émanant des établissements d'enseignement et de recherche français ou étrangers, des laboratoires publics ou privés. 


\title{
ELECTROMAGNETIC PROPERTIES OF THE DAYEM BRIDGE
}

\author{
M. T. LEVINSEN $(*)$
}

\author{
Physics Laboratory I, H. C.-Ørsted Institute, University of Copenhagen, Denmark
}

\begin{abstract}
Résumé. - Les premières mesures faites sur les ponts de Dayem montraient que leur comportement dans un champ hyperfréquence était tout à fait différent de ceux des jonctions Josephson mais d'autres mesures effectuées sur des ponts plus petits ont prouvé que cette différence était due aux dimensions des ponts. Nous avons imaginé un circuit équivalent très simple pour caractériser ces ponts de petites dimensions et avons comparé les résultats obtenus par simulation sur un calculateur analogique aux résultats expérimentaux. A haute température un arrondissement des marches et un supercourant vraisemblablement dû au bruit sont observés. Nous comparons nos résultats à ceux donnés par la théorie d'Ambegaokar et d'Halperin. A basse température un phénomène d'hystérésis se développe, il est vraisemblablement dû à la capacité du pont qui donne aussi naissance à des sous-harmoniques, toutefois la valeur de cette capacité $(1 \mathrm{pF})$ est insuffisante pour rendre compte des sous-harmoniques observés. Les microponts réalisés sont mécaniquement très robustes ce qui n'est pas le cas des autres types de jonctions. Nous nous sommes en outre intéressés aux applications de ces microponts, utilisés comme détecteurs à $10 \mathrm{GHz}$ nous avons trouvé une sensibilité de $9 \times 10^{-14} \mathrm{~W} / \mathrm{Hz}^{-1 / 2}$ et nous avons montré qu'un mélange par effet Josephson était possible. La puissance correspondante aux fréquences de mélange est en bon accord avec les résultats obtenus en simulant le circuit équivalent sur un calculateur analogique. Nous avons montré en observant des marches self-induites dans la caractéristique que les microponts rayonnent.
\end{abstract}

\begin{abstract}
The first measurements on the Dayem bridge showed that the behaviour in an applied microwave field was quite different from that of the Josephson tunnel junction. Measurements on bridges one magnitude smaller later showed this difference to result from the bridge size. A very simple equivalent circuit was furthermore shown to characterize these small bridges. Analog computer calculations on this circuit will be compared to experimental results. At high temperatures a rounding of steps and supercurrent presumably due to noise is observed. A comparison with the theory of Ambegaokar and Halperin will be presented. At low temperatures a hysteresis develops. This may result from the bridge capacitance which also may give rise to subharmonic steps. However, the magnitude ( $1 \mathrm{pF})$ inferred from the hysteresis is too small to account for the subharmonic steps actually observed. In contrast to other types of junctions the bridge is mechanically very robust. We have therefore been interested in the applicability of the bridge. An upper limit to the sensitivity to $10 \mathrm{GHz}$ radiation has been found in terms of $N E P$ to $9 \times 10^{-14} \mathrm{~W} / \sqrt{\mathrm{Hz}}$. Furthermore, we have demonstrated that mixing via the Josephson effect is possible. The power dependence of the sum and difference steps is in fair agreement with analog computer calculations on the equivalent circuit. It has been debated whether the bridge radiates at all. By the observation of a cavity induced step we have now established that bridges do radiate.
\end{abstract}

1. Introduction. - In contrast to all other types of Josephson junctions the Dayem bridge [1] or thin film constriction, is a single metallic structure made either by vacuum evaporation through a mask [1], [2], [3] or by a cutting technique [4]. Although thus inherently stable mechanically it has only recently received attention for application purposes [5].

The first extensive study of the Dayem bridge was published by Dayem and Wiegand in 1967 [3]. Their results on $\mathrm{Al}$, In and $\mathrm{Sn}$ bridges confirmed the results of the earlier works. Applying microwaves of frequencies from $0.2-11 \mathrm{GHz}$ they were able to induce constant voltage steps in the $I-V$ characteristics, but they never saw the Bessel function variation

(*) Present address : Department of Physics, University of Texas at Austin, Austin, Texas 78712. predicted from the Josephson equations. It was therefore concluded that a sinusoidal current-phase relation did not exist for the Dayem bridge and that the synchronization with microwaves comes about in a quite different manner. In order to explain these observations the concept of vortex flow was invoked [1], [3]. Another interesting and puzzling phenomenon observed was the initial increase in supercurrent when microwaves were applied.

The investigation carried out by Dayem and Wiegand thus showed that the Dayem bridge behaved quite differently from a Josephson Tunnel junction. "The microwave effect on the supercurrent of a bridge does not resemble in any way the effect on the Josephson direct current in a Tunnel junction. "

An indication that this was perhaps not the whole truth came in 1970 in an experiment by Fulton and Dynes [6]. They examined the interference pattern 
obtained from applying a dc magnetic field on two bridges in parallel. Extremely close to the transition temperature this interference pattern could be interpreted as resulting from a sinusoidal current-phase relation. Further evidence was presented by Simmonds and Parker [7] who studied the influence of thermal fluctuations on the $I-V$ curve of a bridge close to $T_{\mathrm{c}}$.

That the size of the bridge was the crucial parameter was demonstrated in 1971 by P. E. Gregers-Hansen and the author [4], who succeeded in making Snbridges $0.5 \mu$ wide and $0.2 \mu$ long by use of a scribing technique. These bridges were nearly an order of magnitude smaller than those of the earlier experiments. A careful description of the technique will be given. It was shown that the current-phase relation was indeed sinusoidal down to the lowest temperatures obtainable $(1.6 \mathrm{~K})$ in the experiments if the bridges were small enough.

For these small bridges the variation with microwave field amplitude indeed resembled the Bessel function behaviour calculated for tunnel junctions. The main deviations could be explained by assuming a resistor in parallel with a perfect Josephson element and current sources instead of voltages sources. Calculations on an analog computer on such a circuit could be fitted very nicely to the experimental results.

A theoretical basis for assuming a sinusoidal current-phase relation at least for the dc supercurrent, when the bridge was much shorter than a coherence length, was given by A. Baratoff et al. [8] and P. V. Christiansen et al. [9] using a one-dimensional Ginzburg-Landau approach.

These calculations were later extended by P. E. Gregers-Hansen, G. Fog Pedersen and the author [10] who also found the first order correction term in different limiting situations. By using the boundary conditions for the order parameter and its first derivative, as given by Zaitsev [11] it was shown that an essential condition for a sinusoidal $I(\varphi)$ relation was that the influence of the current on the order parameter in the background films was negligible. The conditions for this requirement were obtained for a Dayem bridge where the cross-sectional area approached zero as well as for the case of $\Lambda$ going to zero (dirty limit). $\Lambda$ is the electron mean free path.

When the film is sufficiently thin the whole film will be in the dirty limit. This situation is the case for several of our bridges. In this case we derived for the current-phase relation the following equation :

$$
\mathrm{I}=\frac{\pi}{4 \mathrm{e}} R_{\mathrm{n}}^{-1} \frac{\Delta^{2}(T)}{k_{\mathrm{B}} T_{\mathrm{c}}} \sin \varphi .
$$

Here $R_{\mathrm{n}}$ is the normal state resistance of the bridge and $\Delta(T)$ the temperature dependent energy gap. Written in this form the relation is identical to the high temperature limit of the current-phase relation obtained by V. Ambegaokar and A. Baratoff [12] from microscopic theory for a Josephson tunnel junction. The only difference lies in the interpretation of the resistance involved which for the tunnel junction is the normal state tunneling resistance. This relation has been experimentally verified by our investigations on microbridges. These results have since been confirmed by other investigators [13], [14].

2. Sample preparation and experimental techniques. - The bridges used in the experiment were made by the method described in references [4] and [10]. A cut was made in the surface of a carefully cleaned glass substrate with a razor blade. The substrate was then etched in $10 \%$ hydrofluoric acid for a few seconds. A 500-2 $000 \AA$ indium or tin film was deposited by vacuum evaporation onto the substrate which was cooled to liquid nitrogen temperature. A second cut was made with a razor blade perpendicular to the first leaving a bridge in the bottom of the groove made by the first cut. The cuts are no longer made by hand, machines having been constructed to do the job. Bridges smaller than $0.5 \times 0.5 \mu \mathrm{m}$ are now routinely obtained. The crucial point in the construction of the machines are the suspension of the razor blades which is done in such a way as to leave them free to align completely to the cutting direction. For the last cut the razor blade is suspended in a thin pianowire in one corner; thus it cuts with its own weight alone. The machines also offer the possibility of making arrays, so far a $2 \times 3$ array has been tested.

The electrical characteristics of the Dayem bridge were measured by drawing the $I-V$ characteristics on an $\mathrm{X}-\mathrm{Y}$ recorder. The current was measured by measuring the voltage drop over a resistor in series with the junction and a nanovolt amplifier was used for measuring the voltage. Two types of cavities were used. A cylindrical tunable cavity and a fixed rectangular with an unloaded lowest resonance at 9.5 GHz. Normally the bridge was placed in the position of optimum coupling which showed up to be the position of strongest electrical field. The temperature was usually determined from the He-vapour pressure. The magnetic field of the earth was compensated and a small magnetic field could be applied in any direction.

3. The simple two fluid model. - 3.1 TEMPERATURE DEPENDENCE OF THE SUPERCURRENT AND MICROWAVE INDUCED STEP STRUCTURE. - As already mentioned the current-phase relation on the supercurrent for a Dayem bridge may be derived from timeindependent Ginzburg-Landau theory yielding the same result as for a tunnel junction as stated in eq. (1). Thus at high temperatures the temperature dependence of the critical current should be linear. That this is indeed the case is shown in figure 1 for a tin bridge. The critical temperature of the bridge region is normally slightly depressed from that of the background thus giving rise to a resistance plateau from 
which we may infer the resistance of the bridge region. Using this we can calculate the slope of the critical current versus temperature from eq. (1). For the bridge in figure 1 we find $0.07 \mu \mathrm{A} / \mathrm{mK}$ compared to the measured value of $0.09 \mu \mathrm{A} / \mathrm{mK}$, well within the uncertainty. Similar agreement is found for nearly all bridges.

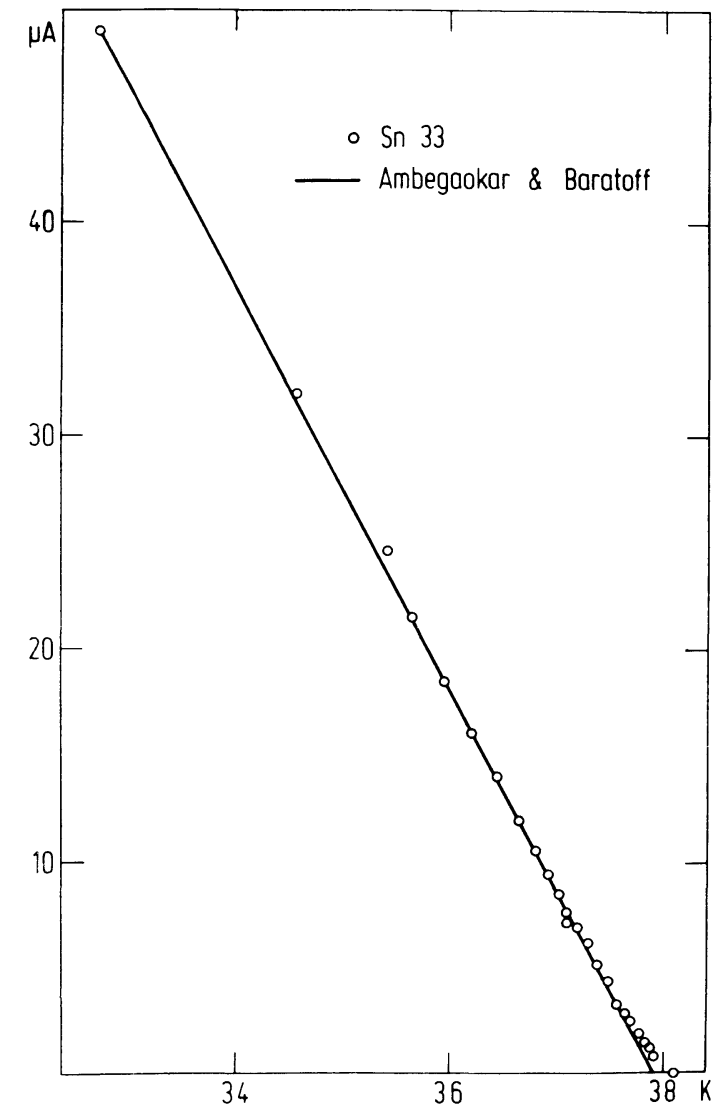

FIG. 1. - Temperature dependence of the critical current of a tin bridge. The solid line represents the theoretical temperature dependence as derived from eq. (1).

For the $I-V$ characteristic no reliable theory exist so far. The simplest approach is that of Aslamasov and Larkin [15] giving as result a two fluid model where the flow of Cooperpairs is described by the Josephson equations while the flow of normal electrons is described by a temperature independent resistor.

$$
\begin{aligned}
& I=\frac{V}{R}+I_{0} \sin \varphi \\
& V=\frac{\hbar}{2 \mathrm{e}} \frac{\partial \varphi}{\partial t} .
\end{aligned}
$$

The $I-V$ curve derived from these equations under the condition that the total current $I$ is constant looks at least qualitatively like that measured for the bridge $\left(V=0\right.$ for $I<I_{0}, V=R \sqrt{I^{2}-I_{0}^{2}}$ for $\left.I \geqslant I_{0}\right)$. However quantitatively there are quite a few deviations.

That the eq. (2) are nevertheless a good practical first approximation for the Dayem bridge shows up when microwaves are applied. In figure 2 are displayed as function of microwave amplitude the heights of the critical current, the first and second of the constant voltage steps which show up in the $I-V$ curve. The solid curves are the result of analogue calculations on the circuit described by eq. (2) where the total current is taken as $I=I_{\mathrm{DC}}+I_{\mathrm{rf}} \sin \omega t$. Considering the very strong distortion of the Bessel functions (as encountered for tunnel junctions) the agreement between experiment and analogue calculation is striking. Thus no matter how serious the deviations in the actual form of the $I-V$ curve seem the Aslamasov-Larkin model is seen to be a good working model for the Dayem bridge. As $I_{0}$ and $\omega$ was known from the experiment the only adjustable parameter was $R$. The value used in the analogue calculation was within $10 \%$ of that inferred from the resistance plateau. This is the case for the whole temperature interval (sometimes as far down as $1.6 \mathrm{~K}$ compared to $T_{\mathrm{c}} \sim 3.8 \mathrm{~K}$ ) where the model worked. Thus the temperature independent bridge resistance seems to be the main determining factor for the properties of the Dayem bridge.

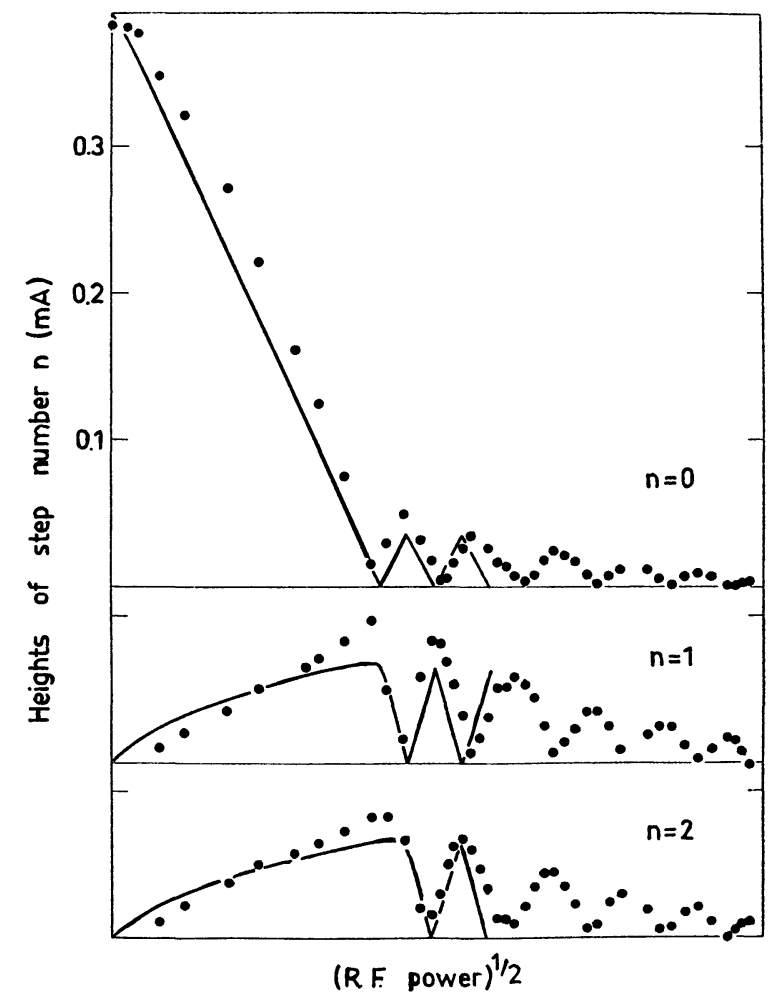

FIG. 2. - The supercurrent and height of the first and second current steps plotted as function of microwave field amplitude for a tin bridge. The solid lines represent the analogue calculations on the basis of eq. (2) using $h v /\left(2 \mathrm{e} R I_{0}\right)=0.09$.

3.2 THE FREQUENCY LIMITS FOR THE APPLICATION OF THE TWO FLUID MODEL. - So far we have described the situation for frequencies around $10 \mathrm{GHz}$. What is the range of frequencies for which the simple 
picture holds. Dahm et al. [16] have shown that the linewidth of Josephson radiation may be written as

$$
\Delta v=\frac{4 \pi k_{\mathrm{B}} T R_{\mathrm{D}}^{2}}{\varphi_{0}^{2} R_{\mathrm{S}}}
$$

for a tunnel junction when $\mathrm{e} V \ll k T$. $R_{\mathrm{D}}$ is the dynamic resistance of the junction and $R_{\mathrm{S}}$ is the static resistance $V / I$ while $\varphi_{0}$ is the flux quantum.

This linewidth is due to thermal noise generated in the junction. When the frequency $v$ of the applied radiation is lowered towards $\Delta v$ the synchronization will break down and the step will become very much blurred out when $v \sim \Delta v$. This formula has been found to be approximately correct for both point contacts and SNS junction although there is no a priori reason why this should be so. Thus there is every reason to believe that it should work for a Dayem bridge. And indeed it does. Let us consider a typical indium bridge : $T \sim 3.3 \mathrm{~K}, I_{\mathrm{c}} \sim 160 \mu \mathrm{A}$, $R_{\mathrm{S}} \sim 5 \mathrm{~m} \Omega$ and $R_{\mathrm{D}} \sim 125 \mathrm{~m} \Omega$ at a voltage corresponding to $500 \mathrm{MHz}$. Inserting in eq. (3) gives

$$
\Delta v \sim 500 \mathrm{MHz} \text {. }
$$

This bridge was investigated at $320 \mathrm{MHz}, 1 \mathrm{GHz}$, $10 \mathrm{GHz}$ and $35 \mathrm{GHz}$. At $320 \mathrm{MHz}$ no steps were observed, at $1 \mathrm{GHz}$ we had clearly resolved steps but alas the equipment did not permit us to measure the power dependence. At $10 \mathrm{GHz}$ the usual distorted Bessel functions were seen. Thus the agreement is very nice but to cite John Clarke [17] « somewhat fortuitous » since the noise-temperature as we shall see seems to be about $30 \mathrm{~K}$.

How about the high frequency limit ? The measurement on the bridge at $35 \mathrm{GHz}$ did show large steps in the $I-V$ curve. However, there was no trace of periodicity and subharmonic steps were prominent. This has been the general trend for most of the few samples investigated at this frequency. Only one indium sample showed clear periodicity, unfortunately it was burned out before the measurement was completed. This behaviour is rather strange especially as we have observed periodicity in step no. 7 and 8 at $10 \mathrm{GHz}$ for samples where no periodicity was observed at $35 \mathrm{GHz}$. That the Josephson ac effect is at work at much higher frequencies is demonstrated by the fact that steps have been observed up to voltages above $2 \Delta / \mathrm{e}$. Up to 60 steps have been observed at $10 \mathrm{GHz}$ in tin bridges at $1.7 \mathrm{~K}$.

3.3 THE EFFECT OF NOISE. - Let us pursue the effect of noise on the behaviour of the bridge. Above we have described the resistance plateau below the transition temperature of the background film and the rounding of the steps. The last phenomenon we have already attributed to thermal noise. Ambegaokar and Halperin [18] have calculated the effect of thermal noise on the $I-V$ curves without applied microwaves for the simple equivalent circuit. A formula was found for $\lim \bar{V} / I$ giving a resistance plateau $I \rightarrow 0$ and some $I-V$ curves were calculated for different values of the parameter $\gamma=\hbar I_{0} / \mathrm{e} k T$ which is the ratio between the binding energy of the junction and the thermal energy. For most of our tin bridges we find noise temperatures ranging from 10 to $1000 \mathrm{~K}$ if the total resistance plateau is explained by noise depression. This does not seem to be likely. For our indium bridges we, however, find a much narrower range, about $30 \mathrm{mK}$. This difference is presumably due to stress stemming from the cut. In figure 3 we have compared an experimental $I-V$ curve for an indium bridge with a theoretical curve taken from reference [18]. It is seen that the overall fit in curve

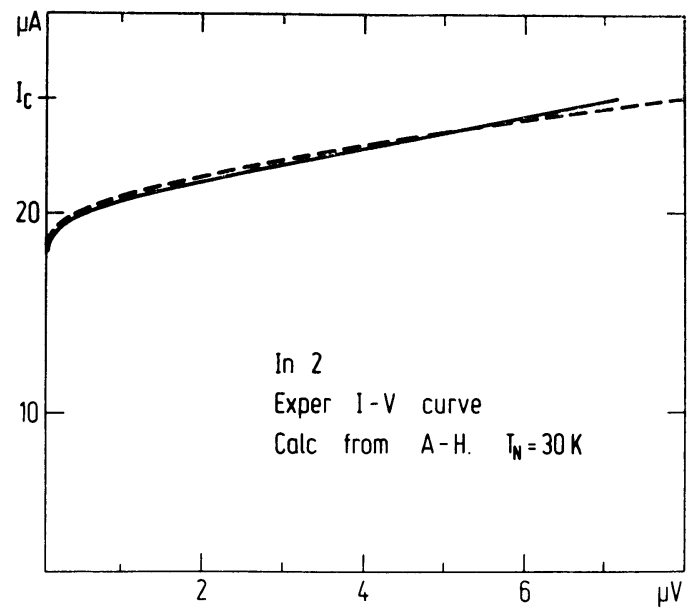

Fig. 3. - Experimental $I-V$ curve (solid line) for an indium bridge about $30 \mathrm{mK}$ below $T_{\mathrm{c}}$ compared to a theoretical $I-V$ curve (dashed line) calculated from the noise theory of reference [18] with $\gamma=20$ corresponding to a noise temperature of $30 \mathrm{~K}$. Theoretical curve taken from reference [18].

form is quite good. Using the plateau resistance we can calculate $I_{0}$ from eq. (1). The value of $\gamma$ obtained from the fit therefore gives us the noise temperature. We find $T_{\mathrm{N}} \sim 30 \mathrm{~K}$ which is a quite likely result as some noise will be coming down the leads from room temperature. Using this temperature we find $\Delta v \sim 5 \mathrm{GHz}$ from eq. (3) which thus seems correct within an order of magnitude. In addition we have investigated the influence of noise on the stepstructure for the simple equivalent circuit using the analogue computer. As noise source was used a white noise generator. The output of the noise generator was adjusted to give a rounding of the $I-V$ curve without microwaves that fitted the observed. The result is shown in figure 4 where we show a set of measured $I-V$ curves for the indium bridge of figure 3 together with the calculated curves. The overall agreement is seen to be quite good although the outer periods in the measurement fall off too fast.

After having given what we believe is the most sensible explanation for the depression of $T_{\mathrm{c}}$ for the bridge region and the rounding of the step-structure let us briefly recall that in the case of a voltage source in series with an inductor instead of the current 


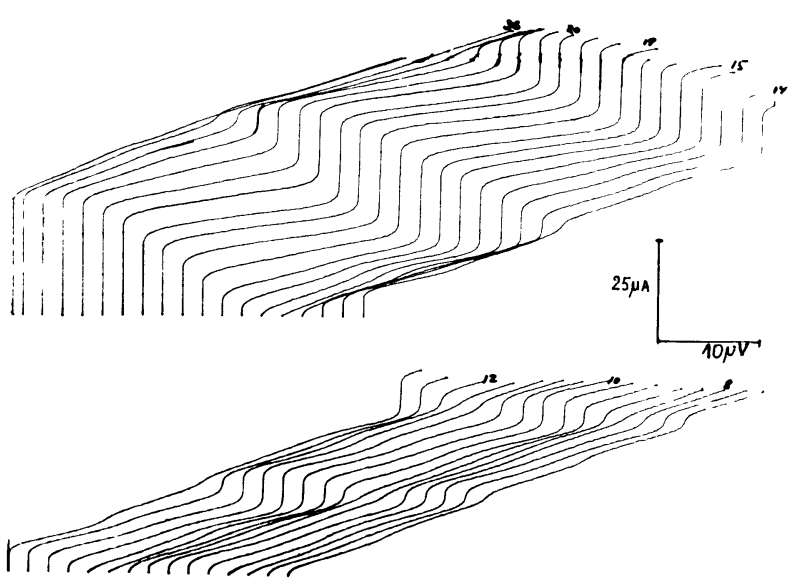

FIG. 4a. - Experimental $I-V$ curves showing rounding of supercurrent and steps presumably due to noise. The curves are measured for an indium bridge (same as Fig. 3) about

$50 \mathrm{mK}$ below $T_{0} . I_{n}=2, T=3.381 \mathrm{~K}, v=9.368 \mathrm{GHz}$.

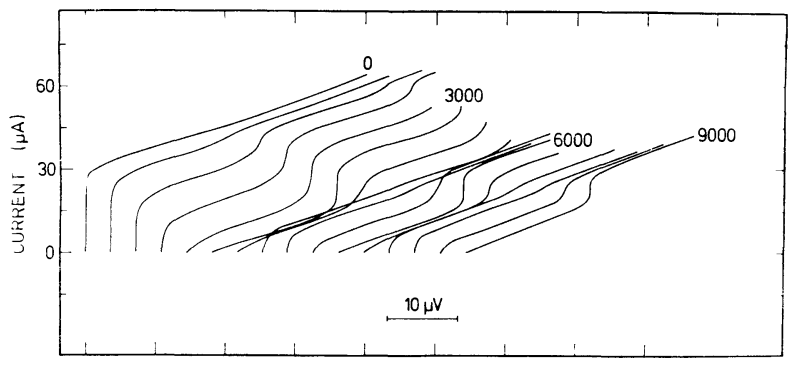

VOLTAGE $(10 \mathrm{uV} / \mathrm{DIv})$

FIG. $4 b$. - I- $V$ curves calculated on the analogue computer from eq. (2). Besides the dc and sine-wave current a white noise current is applied. The supercurrent, resistance, frequency and amount of noise is chosen to fit the experimental curves shown in figure $4 a$.

source we find a similar result. In fact as can be seen from figure $7 b$ of reference [19] the agreement is just as good. Furthermore, the width of the resistance plateau and the depression of the supercurrent with $L \sim 0.6 \mathrm{pH}$ fits as well as what can be calculated from eq. (2) with noise. This illustrates the care which must be taken in choosing the relevant equivalent circuit for a Josephson junction.

4. The hysteresis and subharmonic steps. - As the temperature is lowered from $T_{\mathrm{c}}$ sooner or later all samples develop hysteresis. An example of this is shown in figure 5 for a tin bridge. A further deviation from the predictions of the simple equivalent circuit is the observation of subharmonic steps near $T_{\mathrm{c}}$. The hysteresis has been explained by D. E. McCumber [20] as arising from the capacitance $C$ of the bridge which shunts the Josephson element in parallel with the resistor thus adding a term $C \mathrm{~d} V / \mathrm{d} t$ to the current in eq. (2). Actually most of the capacitance stems from the background film and so will be frequency dependent. The value is expected to be of the order of $1 \mathrm{pF}$. In reference [20] was shown computer calculated $I-V$ curves for this circuit. We have compared the experimental $I-V$ curve of figure 5 with one of the curves of reference [20] shown as the dashed

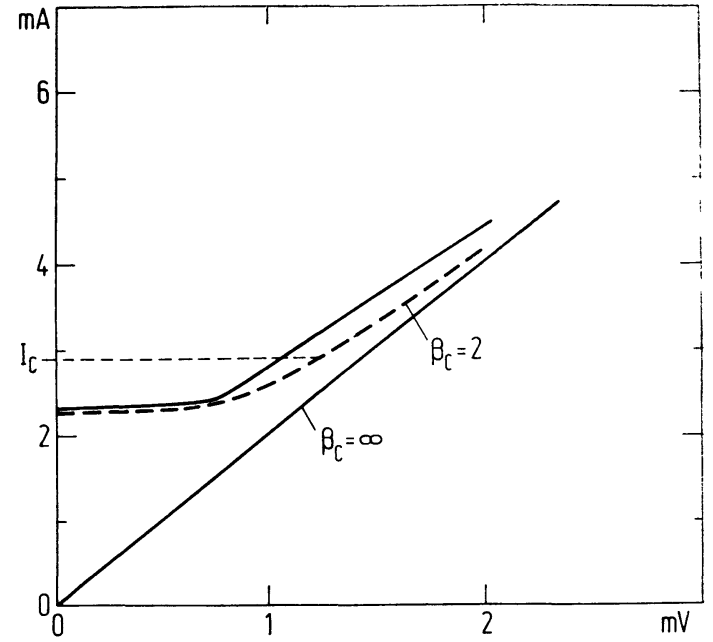

Fig. 5. - I- $V$ curve for a tin bridge at $1.7 \mathrm{~K}$ (solid line) compared to an $I-V$ curve (dashed) calculated for a current biased Josephson element shunted by a resistor and a capacitor. $\beta_{\mathrm{c}}=2 \mathrm{e} I_{0} R^{2} C / \hbar=2$. Theoretical curve taken from reference [20]. The fit gives $C \sim 1 \mathrm{pF}$.

line in figure 5. From the fit we find $C \sim 1 \mathrm{pF}$. Similar results are found for all our bridges. The fact that the experimental curve lies above that of the theoretical curve for voltages higher than $1 \mathrm{mV}$ is possibly connected with the frequency dependence of $C$ but also certainly connected to the subharmonic ( $\Delta$ bump) gap structure and the possible self-induced Dayem effect which is the subject of the following paper by P. E. Gregers-Hansen [21].

Let us now turn to the microwave induced stepstructure. At values of the capacitance where a hysteresis has evolved the analog calculations show that as the rf field is applied the hysteresis is suppressed during the first period and eventually disappear altogether after the first minimum of the supercurrent. That this is in accordance with the experiments is shown in figure 6 . Here are plotted some of the steps for a tin bridge at $T \sim 1.6 \mathrm{~K}$. It is seen that for the lower power levels there is hysteresis in the supercurrent. Then comes a region of some confusion after which the periodicity comes out very clearly. For the higher number steps the periodicity is clear at all power levels. At high power levels the analog calculations give that the periods begin to increase in height. This may be a peculiarity of the analog computer but it is certainly never encountered in the experiments.

The hysteresis from which the capacitance of the bridges is calculated is a low temperature phenomenon. At higher temperatures subharmonic steps are often observed.

Using a spectrum analyzer $\partial \varphi / \partial t$ has been Fourier analyzed on the analogue computer for the capacitor shunted circuit. It was observed that for all current values the amount of higher harmonics in the voltage decreased for increasing capacitance. Thus the capacitance shorts out the higher frequencies as expected. 


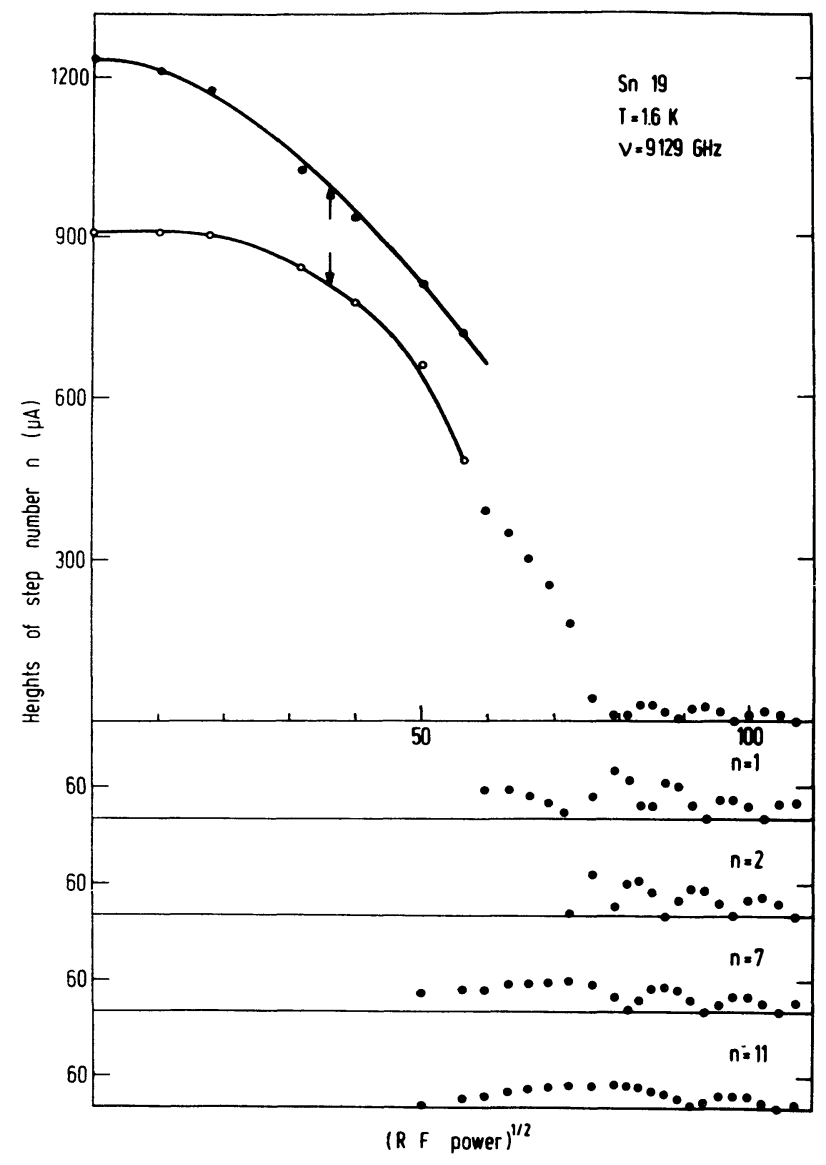

FIG. 6. - Height of supercurrent and some steps as function of microwave field amplitude for a tin bridge at $1.6 \mathrm{~K}$ showing possible influence of a capacitance. $C \sim 1 \mathrm{pF}$. The arrows mark the direction of current change.

However, the capacitance also introduces a phase shift between the current flowing in the resistor and the capacitance. This may explain why subharmonic steps occur in the computed $I-V$ characteristics when an ac current source is applied and $R C_{\omega}$ is about 1 [19], [22]. For $\eta=2$ and $\beta_{\mathrm{c}}=0.625$ a set of $I-V$ characteristics was calculated and subharmonic steps were clearly resolved. In figure 7 are plotted the heights of the supercurrent, the fundamental and some of the subharmonic steps as function of ac current amplitude. The variation of the subharmonic steps is seen to be distorted Bessel functions of type $J_{m}\left(n 2 \mathrm{e} V_{\text {rf }} / \hbar \omega\right)$.

However, the values of $C$ needed to explain the subharmonic steps observed are about 10 times larger than those found from the hysteresis. Indeed the lack of subharmonic steps for the smaller bridges are in good agreement with the values of $C$ obtained from the hysteresis. The subharmonic steps in point contacts and very low resistance tunnel junctions though may well originate in the larger capacitance of these structures. Let us conclude by mentioning that the value of $C$ found from the hysteresis may even be an upper limit as a non-sinusoidal currentphase relation can enhance the hysteresis found for a given capacitance [23].

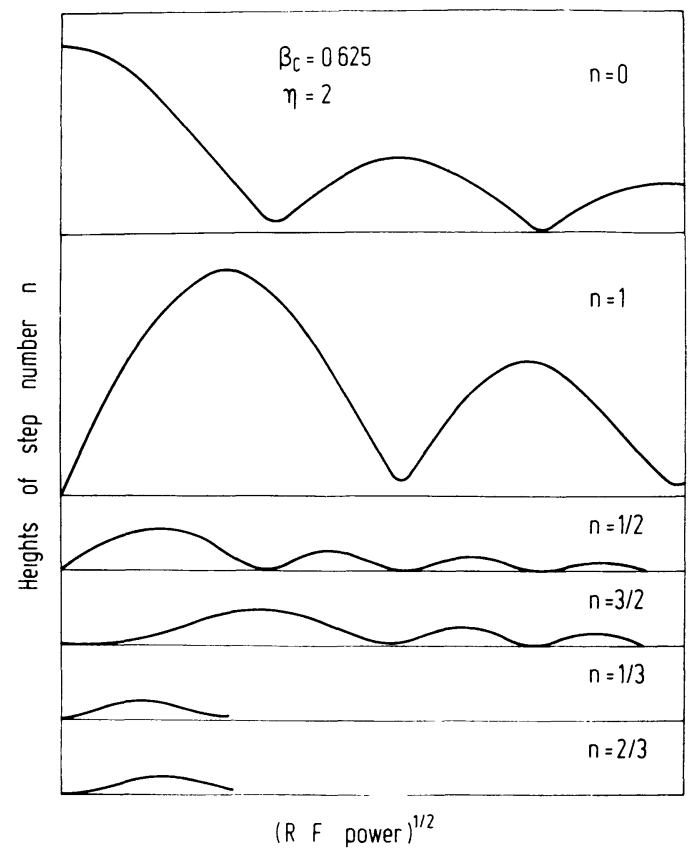

FIG. 7. - Analogue calculations on the capacitively and resistively shunted junction showing variation of steps and subharmonic steps with « microwave current » amplitude.

$$
\begin{array}{llllll}
\beta_{\mathrm{c}} & =2 \mathrm{e} I_{0} & R^{2} & C / \hbar . & \eta
\end{array}=h v /\left(2 \mathrm{e} R I_{0}\right) .
$$

The subharmonic step can, however, be explained by other mechanisms, for instance, by the inclusion of an inductance somewhere in the circuit. The simplest explanation is that the current-phase relation is not sinusoidal [10]. In figure 8 we show the result of an

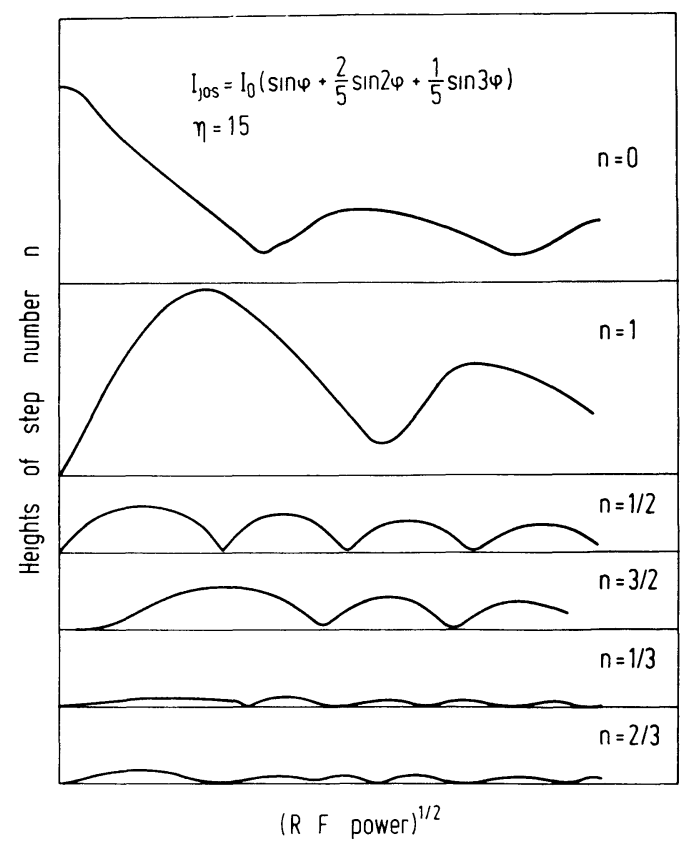

FIG. 8. - Analogue calculation on the simple equivalent circuit with $I_{0} \sin \varphi$ replaced by $\sum I_{n} \sin n \varphi$ showing variation of steps and subharmonic steps with " microwave current " amplitude. It is interesting that already the inclusion of a $\sin 2 \varphi$ term gives rise to $m / 3$ subharmonic steps. 
analogue calculation where in the simple equivalent circuit $I_{0} \sin \varphi$ has been replaced by a sum of $\sin n \varphi$ terms. As seen we again find subharmonic steps varying as distorted Bessel functions of type $J_{n}\left(m 2 \mathrm{e} V_{\mathrm{rf}} / \hbar \omega\right)$. Indeed the two results shown in figures 7 and 8 are very much alike. In both cases when $\hbar \omega / 2$ e $R I_{0} \rightarrow 0$ the subharmonic steps disappear. However, for the latter case the subharmonic steps survive when $\hbar \omega / 2 \mathrm{e} R I_{0} \rightarrow \infty$. In the experiment though they will be quenched by noise. In figure 9 we show an example of the subharmonic steps observed for a tin sample. The $n=m / 3$ series was also observed and had the faster rate expected. Indeed the observed power dependence of the subharmonic steps always resembles that given by the distorted Bessel functions of type $J_{n}\left(m 2 \mathrm{e} V_{\mathrm{rf}} / \hbar \omega\right)$ [10].

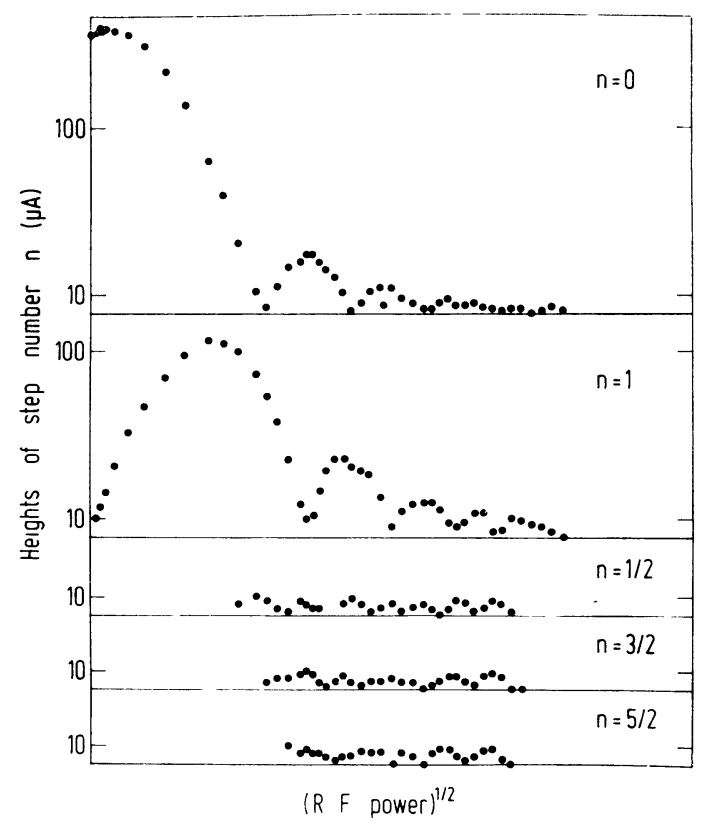

Fig. 9. - Heights of supercurrent, first step and some subharmonic steps as function of microwave field amplitude for an indium bridge. The subharmonic steps are seen to vary in a more or less periodic way.

5. The Dayem effect. - The last deviation from the predictions of eq. (2) we shall discuss is the initial rise in super-current when microwaves are applied, the so-called Dayem effect. This was observed near the transition temperature $\left(T_{\mathrm{c}}-T \lesssim 100 \mathrm{mK}\right)$ for frequencies up to $11 \mathrm{GHz}$ by Dayem and Wiegand [3] and Wyatt et al. [2]. A lower frequency limit for the effect of about $2 \mathrm{GHz}$ was found. This is in agreement with our experiments although the lower limit varies from bridge to bridge and in some cases was above $10 \mathrm{GHz}$. It was furthermore seen that the enhancement had a maximum at a frequency below that corresponding to $2 \Delta$ where pairbreaking would occur. We have extended the measurements to frequencies around $35 \mathrm{GHz}$. In figure 10 we show a comparison of the maximum relative increase obtained at $10 \mathrm{GHz}$ and $34.8 \mathrm{GHz}$ for a tin bridge. As seen the enhancement starts at a much lower temperature in agreement with the requirement $\hbar \omega<2 \Delta$ but reaches a much higher value. Also the enhancement stretches to a much lower temperature; we have observed an enhancement of $8 \%$ at $1.7 \mathrm{~K}$ for a tin sample, i. e., more than $2 \mathrm{~K}$ below $T_{\mathrm{c}}$. This effectively rules out the explanation of the counteracting of thermal fluctuations by the microwaves. To produce a depression of the supercurrent of $8 \% 2 \mathrm{~K}$ below $T_{\mathrm{c}}$ would from the theory of reference [18] require a noise temperature of several thousand degrees Kelvin. In view of the noise temperature of $30 \mathrm{~K}$ presented in section 3.3 this is highly improbable.

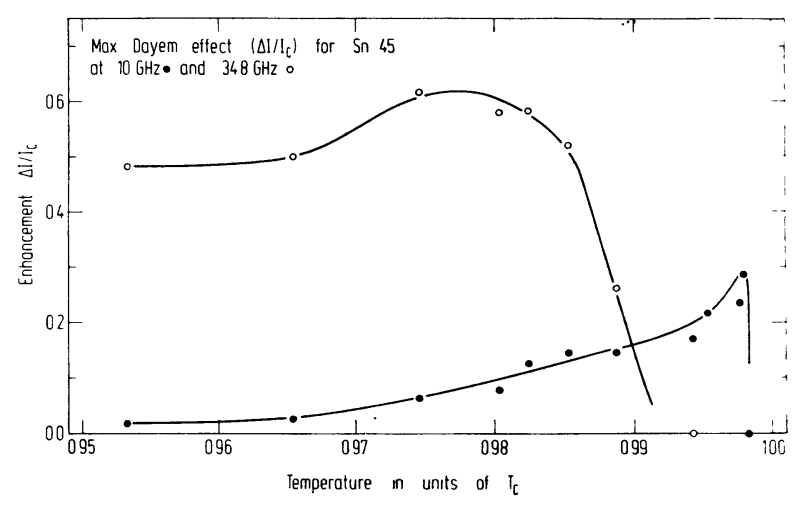

FIG. 10. - Maximum Dayem effect $\Delta I / I_{0}$ as function of reduced temperature $\left(T_{\mathrm{c}}=T_{\mathrm{cBridge}}\right)$ for a tin sample. The arrows mark the temperatures where $h v=2 \Delta$ for $a) 10 \mathrm{GHz}$ and $b$ ) $34.8 \mathrm{GHz}$.

An explanation based on microscopic theory was offered by Eliashberg [24] in 1970 and has since been elaborated [25], [26]. We shall only try to convey the main points of the first paper.

In the BCS theory the energy gap $\Delta$ is determined by the equation

$$
\Delta=g \int_{\Delta}^{\hbar \omega_{D}} \mathrm{~d} \varepsilon \frac{\Delta}{\sqrt{\varepsilon^{2}-\Delta^{2}}}[1-2 n(\varepsilon)] .
$$

Here $\omega_{\mathrm{D}}$ is the Debye frequency ; $\varepsilon$ is the excitation energy of a quasi-particle and $g$ is a measure of the coupling strength. $n(\varepsilon)$ is the distribution function of quasi-particles which in equilibrium is given by the Fermi function. Let us apply an alternating field of a frequency too small for pair breaking to take place. When absorbed by the excitations it will cause the " center of gravity " to be moved to a higher energy, while leaving the total number of excitations unaltered.

The minimum in $1-2 n(\varepsilon)$ will therefore be removed from the region where $\sqrt{\varepsilon^{2}-\Delta^{2}}$ is small, thereby increasing the value of the integral. The result will therefore be an increase in the energy gap. For small values of the field the increase will be proportional to the applied power. A more careful ana- 
lysis gives that there is a minimum frequency $\omega_{\mathrm{c}}(\Delta)$ for the enhancement to take place.

As the effect of the microwaves is equivalent to disregarding $n(\varepsilon)$ in the factor $(1-2 n(\varepsilon))$, there obviously exists an upper limit for the enhancement namely $\Delta(T=0)$. Thus the theory is at least qualitatively in agreement with the experiments, although a precise comparison is impossible due to the geometry of the bridge. The effect should from the theory exist in two-dimensional films. It has, however, never been observed with certainty. It may of course be that a strong spatial variation enhances the effect thus making it observable in Dayem bridges under a certain width. In reference [21] further evidence will be presented that the enhancement of the supercurrent is connected with an enhancement of the gap thus supporting the theory of Eliashberg and coworkers.

6. Experiments with a view on application possibilities. - We shall now turn our attention to some experiments which throw some light on the possibility of practical use of the Dayem bridge as a detector. The experiments discussed will all have bearing on the use of the bridge in the microwave range.

6.1 Sensitivity to $10 \mathrm{GHz}$ Radiation. - Only a few attempts have been made to measure the absolute sensitivity of the bridge as none of the sample holders were designed for this purpose. The measurements therefore give a lower limit to the sensitivity. In one specific case the bridge was biased at a voltage of $\hbar \omega / \mathrm{e}$. In this regime the bridge works as a square law detector since it is the effect on the $I-V$ curve of the decrease in supercurrent that is measured. The experiment gave a sensitivity in terms of $N E P$ of $9 \times 10^{-14} \mathrm{~W} / \sqrt{\mathrm{Hz}}$ at a frequency of $9 \mathrm{GHz}$. This compares to the $5 \times 10^{-15} \mathrm{~W} / \sqrt{\mathrm{Hz}}$ value found by Kanter and Vernon for a point contact at $3 \mathrm{~mm}$. As the $N E P$ value should be proportional to $\omega^{2}$ the sensitivity of the Dayem bridge is thus two orders of magnitude smaller than that of the point contact. However, as mentioned the sensitivity should be considered a lower limit.

6.2 MiXING WITH THE BRIDGE. - When two signals of different frequencies are shone onto a Josephson junction it is well known that not only do we see the fundamental step structures corresponding to the two frequencies but we also observed steps at sum and differences frequencies [27]. Observation of such steps are proof that the junction works as a mixer through the Josephson effect.

In figure 11 we show the result of such a mixing experiment on an indium bridge $70 \mathrm{mK}$ below $T_{\mathrm{c}}$. The power level of a $34.8 \mathrm{GHz}$ signal was kept fixed and the level of a $9.076 \mathrm{GHz}$ signal varied. As seen sum and difference steps do occur. The solid lines represent analogue computer calculations on the

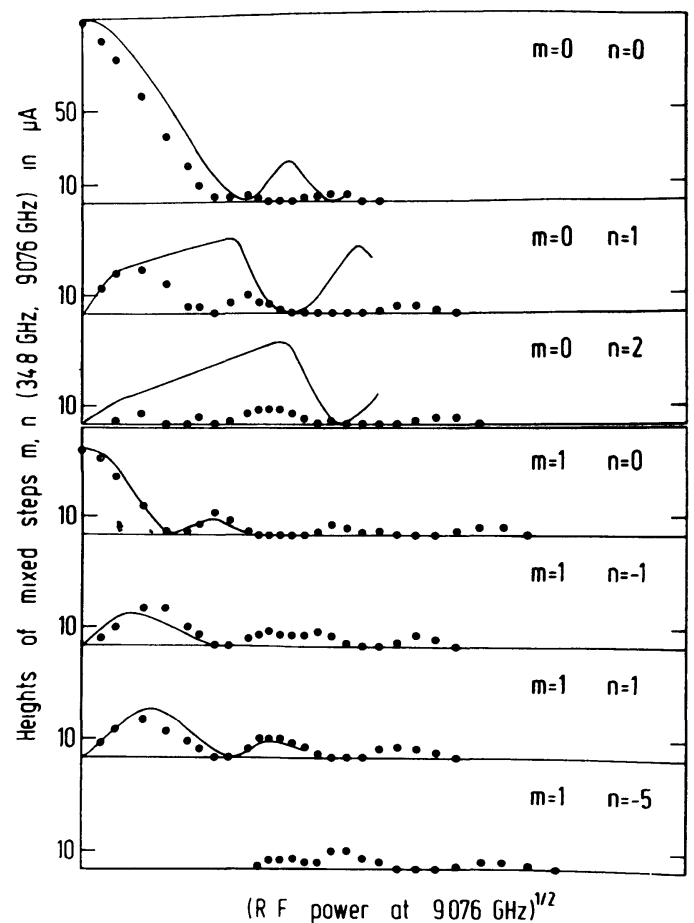

FIG. 11. - Result of mixing experiment on an indium bridge. The power at $34.8 \mathrm{GHz}$ is fixed while that at $9.076 \mathrm{GHz}$ is varied. The solid lines are the result of an analogue calculation on the simple equivalent circuit represented by eq. (2).

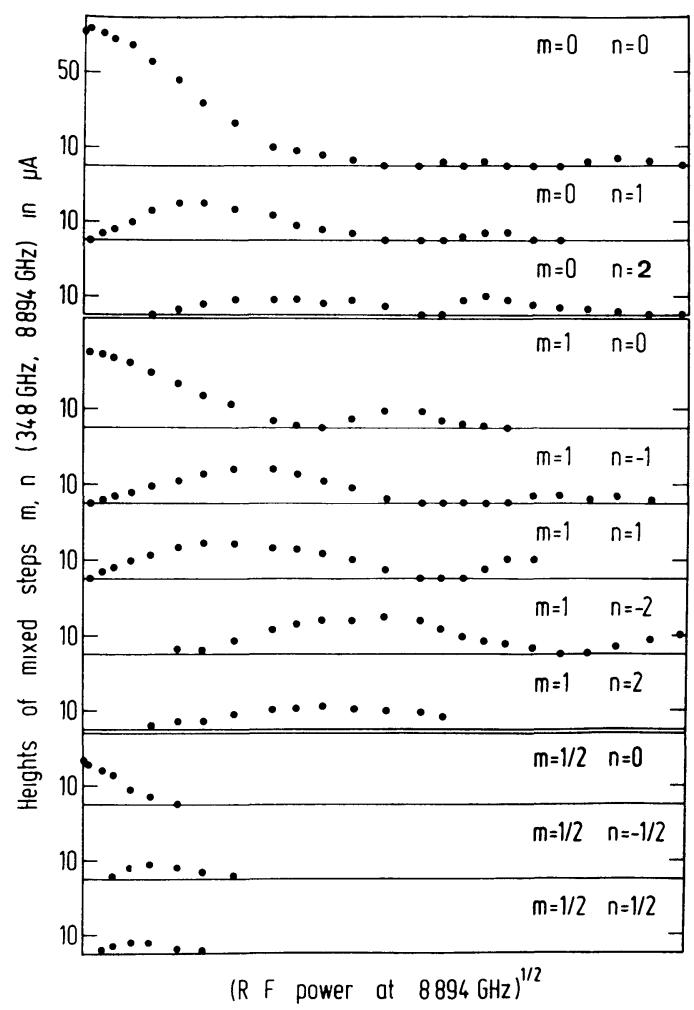

FIG. 12. - Result of mixing experiment on a tin bridge. The power at $34.8 \mathrm{GHz}$ was fixed and that of $8.894 \mathrm{GHz}$ varied. Of special interest is the half step of $34.8 \mathrm{GHz}$ and its satellites spaced $\frac{1}{2} \times 8.894 \mathrm{GHz}$. 
simple equivalent circuit with two frequencies applied. The lack of fit for the first and second $9.076 \mathrm{GHz}$ steps are due to the Dayem effect of the $34.8 \mathrm{GHz}$ radiation which gives rise to hysteresis in this region.

In figure 12 is shown a similar mixing experiment on a tin bridge. Also here the distorted Bessel function behaviour is evident. However, here we also have a half step of the $34.8 \mathrm{GHz}$ radiation and side steps to this with spacing $\left(\omega_{1}-\omega_{2}\right) / 2$. It is also seen that these vary approximately twice as fast as the first $34.8 \mathrm{GHz}$ step and its side steps. This is in agreement with analogue calculations on a system where the current-phase relation is taken as

$$
I_{\mathrm{J}}=I_{1} \sin \varphi+I_{2} \sin 2 \varphi .
$$

This gives the observed half steps with power dependence corresponding to distorted Bessel functions of type

$$
J_{k}\left(2 \times \frac{2 \mathrm{e} V_{1}}{\hbar \omega_{1}}\right) \times J_{l}\left(2 \times \frac{2 \mathrm{eV}}{\hbar \omega_{2}}\right) .
$$

6.3 Cavity Self-Induced SteP. - From eq. (2) we know that when a dc current larger than $I_{0}$ flows through the bridge an ac voltage with frequency components $n \times 2 \mathrm{e} V_{\mathrm{DC}} / h$ will result. When the bridge is enclosed in a cavity the ac voltage may excite cavity resonances. The excited field will then react back on the bridge much like an external field and give rise to a step. The actual form of this step will depend on the circuit elements of the bridge structure involved and on the cavity $Q$.

As shown by Richards and Sterling [28] the junction response to broadband radiation in the case of strong interaction with the cavity is considerably narrowed over what one would expect from the $Q$ value of the cavity. However, the sensitivity to frequencies at or near resonance is enhanced. The existence of a cavity induced step would therefore greatly increase the possibility of practical use of the Dayem bridge as a detector.

The cavity used in the experiment was a rectangular cavity designed to have a lowest resonance at $9.5 \mathrm{GHz}$. This was however shifted downwards by the introduction of the sample holder and glass substrate. The bridge was placed so that the lowest mode $\left(\mathrm{TE}_{101}\right.$ mode) was most likely to be excited.

When observing the cavity induced step the cavity was completely closed. $I-V$ curves were measured at several temperatures whereafter the cavity was opened and $I-V$ curves obtained at the same temperatures. The $I-V$ curves with open cavity fitted exactly the curves obtain with the cavity closed except for a bump protruding around a voltage corresponding to $7.5 \mathrm{GHz}$.

An example of the observed step is shown in figure 13 for an indium bridge of length $0.3 \mu \mathrm{m}$ and width $0.4 \mu \mathrm{m}$. The film thickness was $0.12 \mu \mathrm{m}$. The bridge resistance $R_{\mathrm{b}}$ was about $0.3 \Omega$. The temperature is about $30 \mathrm{mK}$ below $T_{\mathrm{c}}$ which is about $3.4 \mathrm{~K}$. The disappearance of the step, when the cavity was opened, shows the validity of the identification of the step as a cavity induced step. Measurements on other bridges have likewise supported the identification. The bridge was furthermore investigated with $10 \mathrm{GHz}$ microwave radiation and showed microwave induced steps that oscillated in the distorted Bessel function manner in agreement with the description above.

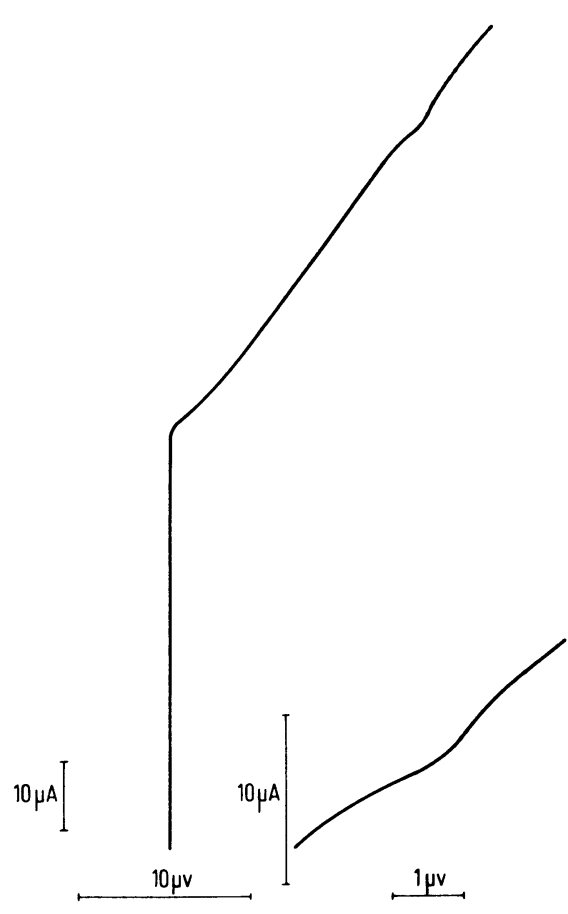

FIg. 13. - I-V curve for an indium bridge showing a cavity self-induced step at a voltage corresponding to $7.5 \mathrm{GHz}$. The step disappeared when the cavity was opened.

The form of the cavity induced step agrees with that calculated by A. Longacre [29] in the heavily damped case thus supporting the validity of the simple equivalent circuit. From Longacre's calculations, it is seen that the high voltage part of the step corresponds to the upper half of the cavity resonance. From this we can calculate the $Q$ value of the system. We find $Q \sim 15$ which is quite agreeable in view of the rather solid sample holder and the glass substrate. The shallow form of the step compared to those of reference [29] is due to this low $Q$ value compare to the $Q$ of 1500 of reference [29].

Acknowledgments. - We wish to thank H. Højgaard Jensen for continued interest and advice during the work. Furthermore we wish to thank P. E. Gregers-Hansen, E. Hendricks, E. P. Pedersen, L. Pedersen, G. Fog. Pedersen and C. J. Sjøstrøm who all have participated in this work. 


\section{References}

[1] Anderson, P. W. and Dayem, A. H., Phys. Rev. Lett. 13 (1964) 195.

[2] Wyatt, A. F. G., Dmitriev, V. M., Moore. W. S. and SheArd, F. W., Phys. Rev. Lett. 16 (1966) 1166.

[3] Dayem, A. H. and Wiegand, J. J., Phys. Rev. 155 (1967) 419.

[4] Gregers-Hansen, P. E. and Levinsen, M. T., Phys. Rev. Lett. 27 (1971) 847.

[5] Goodman, W. L., Hesterman, V. W., Rorden, L. H. and Goree, W. S., Proc. IEEE 61 (1973) 20.

[6] Fulton, T. A. and Dynes, R. C., Phys. Rev. Lett. 25 (1970) 794.

[7] Simmonds, M. and Parker, W. H., Phys. Rev. Lett. 24 (1970) 876

[8] Baratoff, A., Blackburn, T. A. and Schwartz, B. B., Phys. Rev. Lett. 25 (1970) 1096.

Erratum, Phys. Rev. Lett. 25 (1970) 1738.

[9] Christiansen, P. V., Hansen, E. B. and Suøstrøm, C. J., J. Low Temp. Phys. 4 (1971) 349.

[10] Gregers-Hansen, P. E., Levinsen, M. T. and Fog PederSEN, G., J. Low Temp. Phys. 7 (1972) 99.

[11] ZAITSEV, R. D., JETP 23 (1966) 702.

[12] Ambegaokar, V. and Baratoff, A., Phys. Rev. Lett. 10 (1963) 486.

Erratum, Phys. Rev. Lett. 11 (1963) 104.

[13] Song, Yeong-du and Rochlin, G. I., Phys. Rev. Lett. 29 (1972) 419.

[14] Taguchi, I. and Yoshioka, H., J. Phys. Soc. Japan 33 (1972) 92.
[15] Aslamasov, L. G. and Larkin, A. I., JETP Lett. 9 (1969) 87.

[16] Dahm, A. J., Denenstein, A., Langenberg, D. N., ParKER, W. H., Rogovin, D. and Scalapino, D. J., Phys. Rev. Lett. 22 (1969) 1416.

[17] Clarke, J., Phys. Rev. B 4 (1971) 2963.

[18] Ambegaokar, V. and Halperin, B. I., Phys. Rev. Lett. 22 (1969) 1364.

[19] Gregers-Hansen, P. E., Hendricks, E., Levinsen, M. T. and Fog PeDERSen, G., Proc. Appl. S-C. Conf. Annapolis 1972 , p. 597.

[20] McCumber, D. E., J. Appl. Phys. 39 (1968) 3113.

[21] Gregers-Hansen, P. E. and Pickett, G. R., Revue Phys. Appl. 9 (1974) 145.

Gregers-Hansen, P. E., Hendricks, E., Levinsen, M. T. and Pickett, G., Phys. Rev. Lett. 31 no. 8.

[22] Hamilton, C. and Johnson, E. G., Phys. Lett. 41A (1972) 393.

[23] Auracher, F. and Van Duser, T., Appl. Phys. Lett. 21 (1972) 515.

[24] EliashberG, G. M., JETP Lett. 11 (1970) 114.

[25] IVlev, B. I. and Eliashberg, G. M., JETP Lett. 13 (1971) 333.

[26] Ivlev, B. I., Lisitsyn, S. G. and EliashberG, G. M., J. Low Temp. Phys. 10 (1973) 449.

[27] Grimes, C. C. and Shapiro, S., Phys. Rev. 169 (1968) 397.

[28] Richards, P. L. and Sterling, S. A., Appl. Phys. Lett. 14 (1969) 394.

[29] Longacre, A., Proc. 1972 Appl. S-C Conf. Annapolis, p. 712. 